\title{
Using Thermal Stimuli to Enhance Photo-Sharing in Social Media
}

\author{
M. AKAZUE, Glasgow Caledonian University \\ M. HALVEY, University of Strathclyde \\ L. BAILLIE, Heriot-Watt University
}

Limited work has been undertaken to show how the emotive ability of thermal stimuli can be used for interaction purposes. One potential application area is using thermal stimuli to influence emotions in images shared online such as social media platforms. This paper presents a two-part study, which examines how the documented emotive property of thermal stimuli can be applied to enhance social media images. Participants in part-one supplied images from their personal collection or social media profiles, and were asked to augment each image with thermal stimuli based on the emotions they wanted to enhance or reduce. Part-one participants were interviewed to understand the effects they wanted augmented images to have. In part-two, these augmented images were perceived by a different set of participants in a simulated social media interface. Results showed strong agreement between the emotions augmented images were designed to evoke and the emotions they actually evoked as perceived by part-two participants. Participants in part-one selected thermal stimuli augmentation intended to modulate valence and arousal in images as a way of enhancing the realism of the images augmented. Part-two results indicate this was achieved as participants perceived thermal stimuli augmentation reduced valence in negative images and modulated valence and arousal in positive images.

CCS Concepts: • Human-centred Computing $\rightarrow$ Interaction Techniques

General Terms: Design, Experimentation, Human Factors

Additional Key Words and Phrases: Thermal stimuli, stimulation, emotion, visual, valence, arousal, dominance, thermal feedback

\section{INTRODUCTION}

Thermal stimulation has been shown as having the ability to influence emotions in people when presented on their own $[36,42,43]$. Thermal stimuli are made up of parameters such as direction of change (warming and cooling) and amount of change which all contribute to the kind of emotion thermal stimuli can evoke in a perceiver. The affective nature of thermal stimuli is further highlighted when used to augment images. Previous research showed that thermal stimuli can be used to modulate the amount of affect evoked in a perceiver when presented with images [2,14,16,29,32]. Therefore the process of allowing people to augment images shared on social media with thermal stimuli could have the potential to further enhance social media interactions by enabling users to communicate even more affect through the images they share online.

In the world of social media, photo-sharing platforms have consistently experienced a growth in popularity. Photo sharing platforms are used for both archival (backup data) and social (connect and interact with people) purposes [28,44]. Facebook, Snapchat and Instagram are social media platforms that are currently shown to be the fastest growing globally [31,44]. Photo-sharing on social media provides an avenue for the expression and curation of identity in ways that builds and maintains social bonds $[15,20,28]$. Several of these social media photo-sharing platforms (e.g. Flickr, Instagram, Snapchat), allow users to transform the photos they share through the use of picture filters. Research shows that the availability of picture filters in social media platforms is one of the factors which has contributed to their growth, with filtered photos being $21 \%$ more likely to be viewed and $45 \%$ more likely to be commented on by photograph consumers [7]. There is however other factors that contribute to how engaging images shared on social media are perceived. According to Hutto et al. [21] and Bakhshi et al. [7], emotional activation is an important driver of engagement in online social interactions. The inherent emotive nature of images [25] contributes to their ability to drive engagement in online interactions. When discussing emotional activation in images, recent research has shown thermal stimuli as one of the factors capable of influencing emotional activation in images [2,16,29,32].

To explore this potential, in this paper we examine whether social media users can use thermal stimuli augmentation to influence emotions in images shared online in a two-part user study. In part-one we attempt to understand how social media 


\section{Using Thermal Stimuli to Enhance Photo-sharing in Social Media:2 • M. Akazue et al.}

users would use thermal stimuli to influence emotions in the images they share online. We asked participants to provide 10 images ( 5 positive and 5 negative) from their social media profiles or personal collection, which they would be willing to share online. Participants were then asked to choose a preferred thermal stimuli combination for each of the images, based on the emotional effect they wanted the image to have on a perceiver. At the end of this study, each participant was interviewed to understand the emotions they tried to influence in the images augmented, and how satisfied they were in the ability of the resulting augmented images to have the intended effect on perceivers. In part-two of our study a different group of participants were asked to perceive and rate the augmented images created by the participants in part-one. Un-augmented and augmented versions of the images created by part-one participants were rated, in order to determine whether thermal augmentation had the effect they were designed to have. . This two-part study to the best of our knowledge is the first known investigation of how the affective attributes of thermal stimuli could be used to enhance photo-sharing interactions on social media. Overall the entire user study was designed to answer the following research questions:

RQ1: What kind of effect do participants want thermal augmentation to have on negative (low valence/high arousal) social media images?

RQ2: What kind of effect do participants want thermal augmentation to have on positive (high valence/high arousal) social media images?

RQ3: How satisfied are participants in the ability of the augmented images they created to evoke the emotions intended?

RQ4: What effect does the augmented images created have on perceivers?

In the following sections we present background research and provide an overview of evaluation tools and equipment used for the study. We then present the two parts of the user study we conducted and the results obtained. This is followed by a discussion of our findings and conclusions.

\section{BACKGROUND}

\subsection{Thermal Stimulation and Effect on Emotions}

Thermal stimuli combinations made up of different thermal stimuli parameters have been shown to have the ability to evoke emotions in people when perceived on their own [27,35-37,41-43]. Research has shown distinct thermal stimuli changes within the temperature range of $26^{\circ} \mathrm{C}$ and $38^{\circ} \mathrm{C}$ to be detectible by perceivers, with higher intensities being more perceivable and less comfortable than lower intensities [17,43]. Thermal stimuli rates of changes (ROC) have also been shown to be perceptually different [22]. Wilson et al. [43] in a study investigating thermal stimuli perception showed that slower ROC's of $1^{\circ} \mathrm{C} / \mathrm{s}$ ROC's were perceivable while faster ROC's of $3^{\circ} \mathrm{C} / \mathrm{s}$ have better detection rates. Akazue et al. [2] in a different study investigating the affective influence of thermal stimuli on images showed that when thermal stimuli is used to augment images $1{ }^{\circ} \mathrm{C} / \mathrm{s}$ and $3{ }^{\circ} \mathrm{C} / \mathrm{s}$ ROC's are perceptually similar. When considering the utility of warm and cool stimuli in thermal feedback design, Wilson et al. [43] found that both warming and cooling temperatures were perceptually different with warm stimuli perceived as less comfortable and more intense than cool stimuli. Research by Lee et al. [27] as part of their investigation into using thermal stimuli as an expression medium found that the effect of thermal stimuli was stronger on a visceral level than on a cognitive level, when used to communicate emotion. Research by Wilson et al. [41,42] however showed that thermal still has a more definite effect on a cognitive level when presented on its own. Their results showed $2^{\circ} \mathrm{C}$, $4^{\circ} \mathrm{C}$ and $6^{\circ} \mathrm{C}$ thermal stimuli intensities changing from cool to warm increased feelings of valence, while the same intensities changing from warm to cool reduced valence [42]. A contrasting result was reported by Salminen et al. [35,36] in their investigation of the effect of thermal stimuli on emotions. Their results show warm stimuli was rated as being more pleasant and arousing than cool stimuli. Wilson et al. [41,42] and Salminen et al. [35,36] however used different procedures for their respective experiments and the thermal stimuli combinations they evaluated also had different rates of change.

In the investigation of factors that affects thermal stimuli perception, some external factors, have been shown to contribute to how well thermal stimuli are perceived. Environmental factors such as extreme ambient temperature and humidity conditions (humidity $<30 \%$ and $>90 \%$ ) were shown to have an influence on detection rate, time to detection, and subjective perceived comfort of thermal stimuli [18,40]. In the presence of clothing, Halvey et al. [19] showed that even though perceived thermal stimuli feels more comfortable than when applied directly to the skin, higher thermal stimuli intensities are needed for 
Using Thermal Stimuli to Enhance Photo-sharing in Social Media:3 • M. Akazue et al.

detection to take place. Existing skin temperature which is the position the skin temperature falls within the neutral zone $\left(-28^{\circ} \mathrm{C}\right.$ and $\left.-40^{\circ} \mathrm{C}\right)$ [22] and thermal stimuli delivery location are also capable of affecting thermal stimuli perception. Thus creating the need to adapt the skin to a neutral stimulus before thermal stimuli presentation in order to maximize perception [24].

When thermal stimuli is used to augment images, Nakashige et al. [32] found that in limited scenarios, images of warm food presented with warming thermal stimuli intensities felt more delicious to participants in their study. Results from Halvey et al. [16] also showed that warm stimuli elevated feelings of arousal and valence in music and images. While this is a good indication of the ability of thermal stimuli to modulate affect, the effect reported in their study was limited to a subset of handpicked images. Lochtefeld et al. [29] in their study evaluating the effect of ambient feedback (ambient light and thermal stimulation) on images viewed on a tablet showed that thermal stimuli contributed to enriching the user experience for mobile media consumption. Although the findings of Lochtefeld et al. [29] highlight the affective possibility that can be afforded by augmenting images on a mobile device with thermal stimuli, the effect they reported was not entirely due to thermal augmentation, also the handpicked images they augmented had no pre-identified emotional property. Findings by Akazue et al. [2] found that simultaneous presentation of thermal stimuli while an image is being displayed on a screen can be used to enhance/reduce affect in the image. The images Akazue et al. [2] augmented were standardised pictures for measuring emotion and attention [25]. Their results showed that low valence (negative) images can be made more negative by augmenting them with warming $38^{\circ} \mathrm{C}$ intensities or less negative by augmenting them with cooling $26^{\circ} \mathrm{C}$ intensities. While $29^{\circ} \mathrm{C}$ and $35^{\circ} \mathrm{C}$ cooling and warming intensities were shown to be effective for increasing valence and arousal in high valence (positive) images.

\subsection{Motivation for Online Image Sharing}

There has been substantial research investigating the motivation for sharing images on social media and how to promote social interactions using pictures [15,20,26]. According to Gulotta et al. [15] digital photographs are generally seen as online identity-building resources, which provide greater opportunities for self-expression than traditional photography groups $[10,15,20]$. Online photo sharing platforms are also seen as an avenue for self-presentation - an opportunity for people to use pictures to present themselves in a way they want to be perceived by others [20]. Pictures are powerful impressions that can have layers of meaning and can often evoke a wide range of emotions in people [30]. There is therefore a need to find ways to allow people to have some control over how the images they share are perceived, by influencing the kind of emotions evoked by such images.

\subsection{Influencing Emotions in Online Image Sharing}

The use of graphical filters to enhance images has been shown to be one way of allowing social media users an influence on the affect their images have when shared online, with filtered Flickr images being more likely to be viewed and commented on [7]. Results from Bakshi et al. [7] showed that even though most of their study participants enjoyed applying filters on their photos, a few participants did not use filters on their photos mainly for self-representation reasons, such as the artificial look of filters on their photos. The use of thermal stimuli to augment images shared online provides a way of allowing users to influence affect in images [2,16,32,36,42], without affecting the visual composition of the image. Previous work examining the affective attributes of thermal stimuli has mainly focused on determining if thermal stimuli augmentation does have an effect on emotions in images [16,29,32] and identifying the effect of individual thermal stimuli parameters on images with varying emotional properties $[2,16]$. The two-part study presented in this paper focused on investigating the potential of allowing users to influence emotions in social media images using thermal stimuli combinations with known affective influence on images.

\section{USER STUDY PART-ONE: AUGMENTING SOCIAL MEDIA IMAGES WITH THERMAL STIMULI}

Our user study was designed to investigate whether social media users are able to harness the affective properties of thermal stimuli augmentation to influence the emotions they intend in the images shared online, as a way of enhancing online photosharing interactions. To answer our research questions (Section 1, 'INTRODUCTION'), we divided the study into two parts. 
Using Thermal Stimuli to Enhance Photo-sharing in Social Media:4 - M. Akazue et al.

Part-one examined the emotions social media users want to influence when creating augmented images and part-two investigated whether the augmented images created had the desired effect on perceivers.

In this first part of our user study, we explored whether thermal stimuli combinations found to be effective in influencing affect in previous work $[2,16,42,43]$ can be used by participants to influence specific emotions in social media images. For example previous research by $[1,2]$ has shown thermal stimuli $\left(26^{\circ} \mathrm{C}, 29^{\circ} \mathrm{C}, 35^{\circ} \mathrm{C}, 38^{\circ} \mathrm{C}\right)$ as especially having the highest effect in modulating affect in very negative (low valence/high arousal) images and very positive (high valence/high arousal) images. The ability of thermal stimuli augmentation to enhance/reduce affect in images was also shown to be most effective when thermal augmentation is presented simultaneously with the image $[1,2,16,32]$. The images used by $[1,2,16]$ were however obtained from a standardised picture library, while no information was provided about how the images used by [32] were sourced. The user study conducted for this paper was designed to utilise images provided by social media users (Fig. 1). Investigations of the emotional property of images currently shared on social media profiles, shows the reluctance of some social media users to share negative events (low valence/high arousal) with their social network as against positive ones (high valence/arousal) due to self-presentation concerns [11]. However a sizeable number of people still share unconventional negative (low valence) images online. Research by Andalibi et al. [4] found that a search of low valence terms on Instagram (e.g. \#depressed) reveals millions of images. According to Gulotta et al. [15] this could be as a result of self-advocacy, a concept where people advocate for the right to be themselves. The increasing popularity of photo-sharing platforms (e.g. Snapchat) that defaults towards ephemerality by automatically deleting content once a receiver views it, also has the potential to significantly reduce the self-presentation concerns associated with automatic archiving of images.

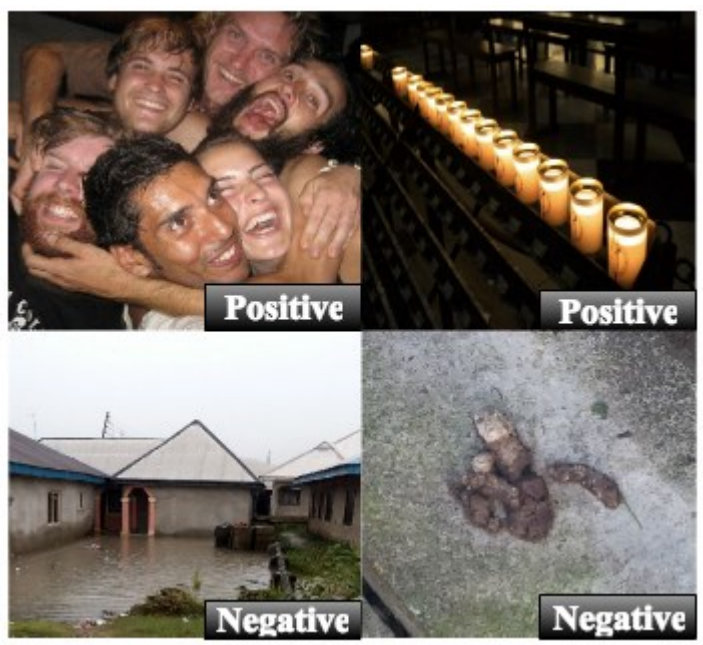

Fig. 1. Sample images provided by participants, showing positive and negative images.

Since thermal stimuli augmentation has been shown to be most effective in influencing emotions in very positive (high valence/arousal) and very negative images (low valence/arousal) [2], we requested participants to provide images corresponding to those emotion categories.

Also we requested participants to provide images from only two emotion categories (positive and negative), because the effect images have on emotions can be subjective, as a result, some of the images provided by study participants and classified as either positive or negative, may be perceived as evoking other emotions by other participants. Participants were told the images they provide could either be images already shared on their social media profiles, or images from their personal collection, which they would be willing to share online. Participants were asked to provide a total of 10 images, 5 images perceived as evoking negative emotions and 5 images perceived as evoking positive emotions (see Fig. 1). In order for us to ensure participants understood the emotional properties of the images they were asked to provide, in the recruitment email we described negative images as images that evoke unhappiness/embarrassment or annoyance, while positive images were described as images that evoke happiness, pleasure, satisfaction or contentment [25]. 
Using Thermal Stimuli to Enhance Photo-sharing in Social Media:5 • M. Akazue et al.

\subsection{Equipment}

Two $2 \mathrm{~cm}^{2}$ Peltier modules were used as the thermal stimuli source in the two user studies (Fig. 2). Similar Peltier stimulators have been used in previous studies investigating thermal stimuli perception [2,18,41-43]. Each Peltier stimulator allows heating and cooling, and is Bluetooth-controlled. Both Peltier stimulators allowed temperatures to be set anywhere within the range of $-20^{\circ} \mathrm{C}$ to $45^{\circ} \mathrm{C}$ accurate to $0.1^{\circ} \mathrm{C}$. For the two parts of the study, images were presented on a laptop screen with 15.6 inch display. All studies were conducted in a quiet lab.

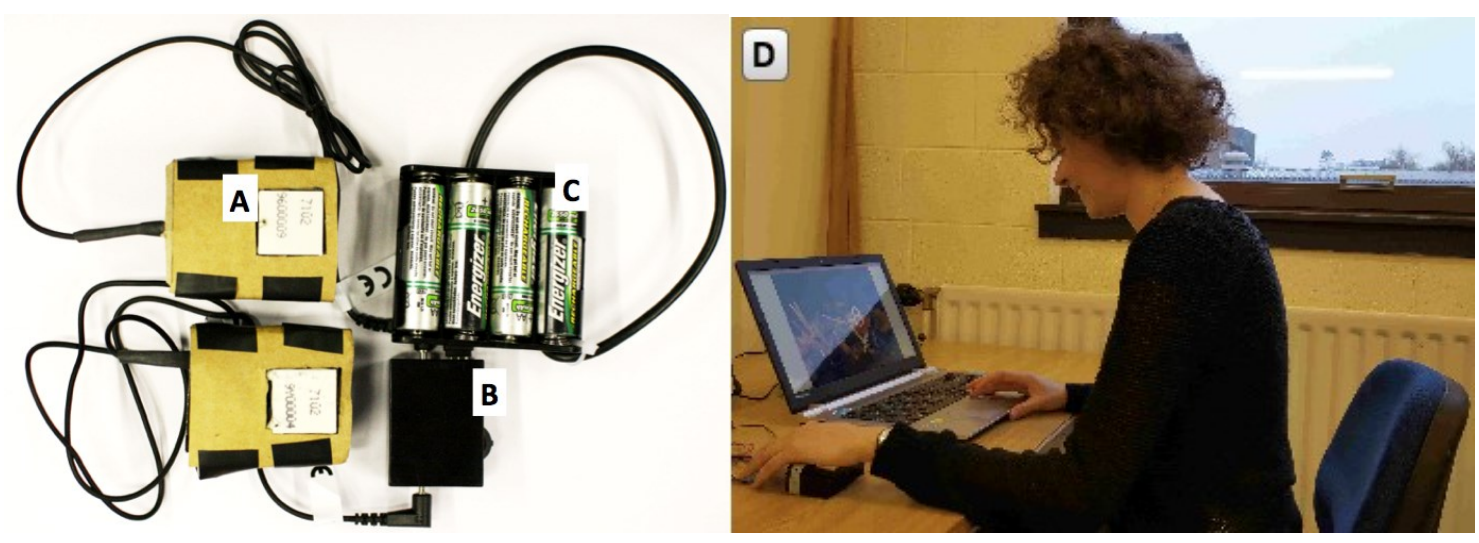

Fig. 2. Peltier stimulators (A), Bluetooth controller (B), Battery pack (C), Study setup (D)

\subsection{Thermal Stimuli Combinations}

Four stimuli combinations made up of thermal stimuli parameters shown to be effective in previous work [2,16,43] for influencing affect in images were used in our user study. In both parts of our study, thermal augmentation was presented to the thenar eminence (bulbous area of the palm) of the non-dominant hand, which has been shown to be the optimal hand location for thermal stimuli delivery [43], others also used a similar procedure [2,16,36,42]. It is also a natural location for thermal stimuli presentation if the thermal presentation device is embedded on a hand-held mobile device [40] or in a mouse [32].

Table 1. Thermal stimuli combinations (represented with labels) used in user-study part-one and their potential emotive properties

\begin{tabular}{ll}
\hline \multicolumn{1}{c}{ Thermal Labels } & \multicolumn{1}{c}{ Attributes } \\
\hline Very cool $\left(\mathbf{2 6}^{\circ} \mathbf{C}\right)$ & $\begin{array}{l}\text { Increase valence in negative (low valence) images } \\
\text { Reduce valence and arousal in positive (high valence/arousal) } \\
\text { images }\end{array}$ \\
\hline Cool $\left(\mathbf{2 9}^{\circ} \mathbf{C}\right)$ & $\begin{array}{l}\text { Increase valence in negative (low valence) images } \\
\text { Increase valence and arousal in positive (high valence) images }\end{array}$ \\
\hline Warm (35 $\left.{ }^{\circ} \mathbf{C}\right)$ & $\begin{array}{l}\text { Increase arousal in negative (low arousal) images } \\
\text { Increase valence and arousal in positive (high valence) images } \\
\text { Very warm }\left(\mathbf{3 8}^{\circ} \mathbf{C}\right)\end{array}$ \\
& $\begin{array}{l}\text { Reduce valence and arousal in positive (high valence/arousal) } \\
\text { images }\end{array}$ \\
\hline
\end{tabular}

All thermal stimuli combinations changed from a neutral point of $32^{\circ} \mathrm{C}$ which lies well within the defined neutral zone for the skin following the procedure of $[17,43]$ at a fast rate of change of $3^{\circ} \mathrm{C} / \mathrm{s}[2,16,43]$. The thermal combinations used were made up of the following thermal stimuli parameters:

(1) One neutral stimulus of $32^{\circ} \mathrm{C}$, which lies well within the defined neutral zone for the skin, at which there is no discernible thermal sensation as shown by $[2,17,43]$. 
(2) Two directions of changes, following the approach of $[2,16,40,42,43]$. Temperatures changed from neutral, resulting in warming and cooling.

(3) Two stimuli intensities of $3^{\circ} \mathrm{C}$ and $6^{\circ} \mathrm{C}$ changing from neutral, which has been shown to have a significant effect on emotions evoked by images [2,16,36,43], giving a total of 4 intensities $\left(26^{\circ} \mathrm{C}, 29^{\circ} \mathrm{C}, 35^{\circ} \mathrm{C}\right.$, and $\left.38^{\circ} \mathrm{C}\right)$.

(4) One rate of change (ROC) of $3^{\circ} \mathrm{C} / \mathrm{s}$, which has been shown to have a similar effect on the emotional perception of images as $1^{\circ} \mathrm{C} / \mathrm{s}$ ROC $[2,23]$.

The combination of parameters above resulted in the 4 thermal stimuli labels used for this study (Table 1). The thermal stimuli combinations and their effect on images as shown by Akazue et al. [2] are shown in Table 1.

\subsection{Study Procedure}

An information sheet containing details about the study was emailed out to participants before the study. On the day of the study prior to beginning the experiment, participants were made aware of the potential emotive properties of the thermal stimuli combinations used as shown by [2]. Similar to how images shared on social media are perceived by the social media contacts of different individuals, all participants in part-one were told the augmented images they create will be perceived and rated by other users. The study started with a training/familiarisation phase, and was conducted using a 15.6-inch screen laptop. During the training phase on the study day, participants were allotted 5 minutes to perceive and familiarise themselves with the thermal stimuli combinations available for use in the study and the effects of individual combinations. Buttons with labels corresponding to the thermal stimuli combinations (very cool, cool, warm, very warm) were displayed on screen for participants to select and pair with images to be augmented. Although participants were able to refer back to the emotive properties of each thermal stimuli combination, none of the 10 participants referred back to them after beginning the experiment. Clicking on any of the buttons triggered the presentation of the stimuli combination it represents for 6 seconds, after which the temperature returned to neutral (See Fig. 3).
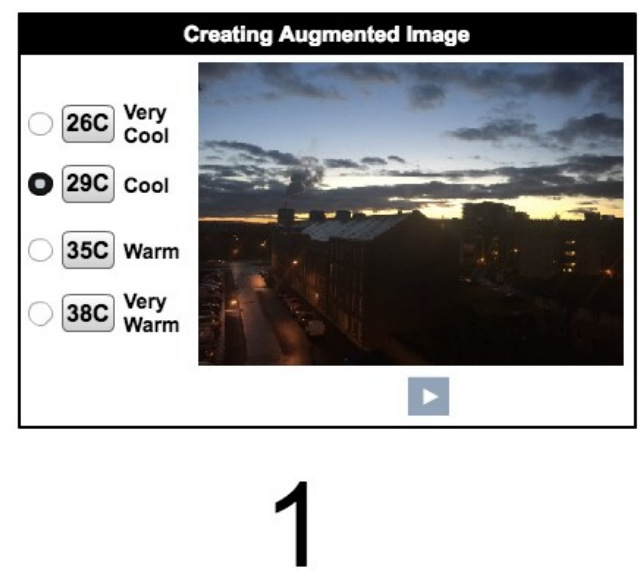
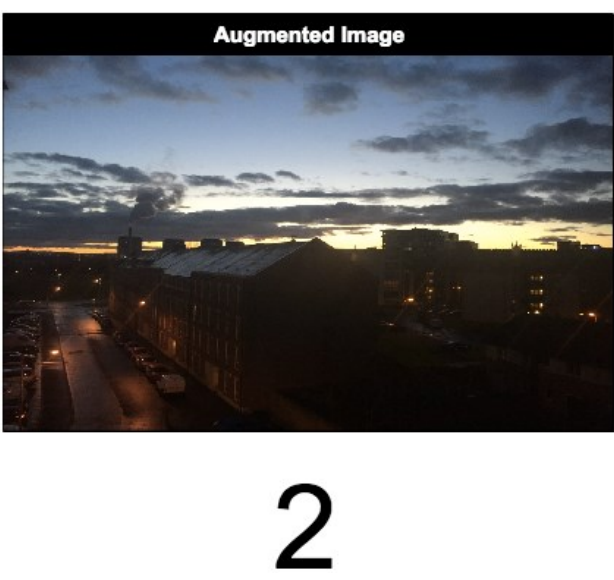

Fig. 3. The screen for pairing images with preferred thermal stimuli combination (1); the slideshow presenting the augmented images created by participants (2).

After the training phase, the 10 images provided by each participant were presented individually in a random order for participants to augment with thermal stimuli. Participants chose a thermal stimuli combination for each image, based on the effect they wanted the augmented image to have on perceivers. Each participant created a total of 10 augmented images, 5 category 1 (positive) images and 5 category 2 (negative) images. After each participant finished pairing images with thermal stimuli, there was a slideshow displaying each image augmented, together with the thermal stimuli selected for that image. Study participants perceived the thermal combinations by clicking the buttons as many times as they wanted when creating augmented images, but were not able to change their selections during the slideshow. Participants were interviewed after they finished creating augmented images to understand the effects they expect thermal stimuli to have on the images. The study lasted for approximately 30 minutes, and all participants were presented with a $£ 10$ Amazon voucher on completion. Russell [33] showed that emotional states can be represented by combinations of valence and arousal. However in the first part of the study, participants were not explicitly asked to consider the effect they want the augmented images created to 


\section{Using Thermal Stimuli to Enhance Photo-sharing in Social Media:7 • M. Akazue et al.}

have on feelings of arousal in perceivers. This is because according to Tellegen [38], high positive and high negative affect both involve high levels of arousal. Bradley et al. [9] also showed that increases in either pleasure or displeasure tend to produce increases in ratings of arousal. This indicates that if a participant is able to successfully use thermal stimuli augmentation to increase or reduce valence in positive/negative images, this will be simultaneously accompanied by an increase in feelings of arousal.

\subsection{Participants}

Study recruitment was open to participants above the age of 18 willing to share positive and negative images from their personal or social media profiles. Only male participants responded to recruitment advertisement and a total of 10 participants were recruited for the study. Participants' ages ranged from 24 to 40, with a mean age of 28 . According to the details filled out on the pre-evaluation questionnaire, 9 out of the 10 participants were active social media users (Snapchat, Instagram, and Facebook), and 6 out of the 10 participants had already shared at least $60 \%$ of the images they provided on their Facebook profiles. Findings by Sabatinelli et al. [34] showed that male and females have similar emotional reactions to the images in the International Affective Picture System (IAPS) library, which contained images with the same emotional properties as those provided by our participants. Akazue et al. [2] also reported similar results; they only found a gender difference for feelings of dominance after images had been augmented with thermal stimuli, with augmented images making females feel more dominant than males. Furthermore when a thermal stimuli combination is presented on its own, males and females have also been shown to have similar thermal stimulus thresholds [13].

\subsection{Data Gathering Method}

An interview was conducted at the end of the experiment, to obtain subjective feedback. The interview was designed to help provide answers to research questions 1-3 (see Section 1, 'INTRODUCTION'). Participants were asked a series of openended questions, and the researcher took notes of responses as well as recording them on an audio recorder. Oral recordings were later transcribed after the completion of the study and analysed (See Section 4). Interview questions were:

(1) Why did you want to augment the negative (category 2) images?

(2) Which emotion did you want to enhance/reduce in negative images?

(3) Why did you want to augment the positive (category 1) images?

(4) Which emotion did you want to enhance/reduce in the positive images?

(5) How satisfied are you with the effect your thermal combination of choice had on each of the images?

Interview questions 1 and 3 were designed to understand the effect participants wanted augmented images to have on a perceiver, and act as a lead into questions 2 and 4, as part of a suite of questions to help identify the emotions participants wanted to influence using the available thermal stimuli combinations.

\section{USER STUDY PART-ONE RESULTS}

Interviews were transcribed and analysed using an adapted version of Constant Comparative Method [5,12]. Incidents were first coded into multiple categories, after which related categories were combined to form 4 higher-level concepts, which are outlined below.

\subsection{Re-creating emotive experiences}

Seven out of ten participants wanted to augment category 2 (unpleasant/negative) images mainly to reflect the physical emotions they felt when capturing the image. The emotions participants felt when capturing an image were influenced by external factors such as the ambient/atmospheric temperature of the location where the picture was captured, time of year or their stress/arousal levels. P5 commented "Actually when I chose a temperature, I chose a temperature related to the ambient temperature of when I captured the pictures. For example if it was warm, it reminded me it was a warm area and I chose a warm temperature to make myself sympathise with that photo". P1 also suggested that their stress/arousal levels while capturing a particular image also contributed to the emotion they wanted to express when using thermal stimuli to augment category 2 images. "Umm... so some unpleasant pictures like for example I had a building, a very old creepy building, in that case I would want to enhance my feeling, I would want a cold kind of temperature if I see that image, so that helped me actually feel how... You know I would normally associate cold with you know creepy... you know frightened situation". 


\section{Using Thermal Stimuli to Enhance Photo-sharing in Social Media:8 - M. Akazue et al.}

Another reason participants provided for wanting to augment unpleasant images was to express the negative emotions they felt as a result of the subject of the image they provided. According to P3 "The unpleasant images that portray, should I say my sad emotions, like how I feel about those images...they are images that cast my mind on bad past experiences and they are not positive in themselves definitely thermal stimuli helps express how I feel about those images". The above results shows that thermal augmentation allowed users to re-create the emotive experiences they had when they captured category 2 (negative) images

\subsection{Increasing Realism}

Seven out of ten participants wanted to use thermal stimuli to increase how realistic the images they were sharing felt, thus further reducing feelings of valence in negative images and increasing valence and arousal in positive images. P7 noted that, "For the negative picture I just selected a temperature to make the images more realistic, not more pleasant. I believe it makes the pictures become more negative because I like to see the facts not just lie to myself". According to P9 "I wanted them (category 1 images) to feel more pleasant". Three out of ten participants wanted to use thermal augmentation to increase feelings of valence in negative images; they tried to do this in order to suppress the unpleasant memories the images evoked. P4: "I was trying to make them feel more pleasant, I wanted to reduce the negativity, that feeling that I have from looking at the picture, so I tried to use something like... a little bit cool to feel a little bit better". This investigation of why participants wanted to augment images showed that users attempted to use thermal augmentation to increase the realism of images they had captured.

\subsection{Manipulating Valence}

Moderate warming and cooling temperature intensities $\left(29^{\circ} \mathrm{C} \mathrm{\&} 35^{\circ} \mathrm{C}\right)$ maintained their ability to increase valence and arousal in all image emotion categories. According to $\mathrm{P} 4$, they tried to increase valence in some of the negative images by using the $29^{\circ} \mathrm{C}$ thermal combination "For some of the negative pictures, I tried to use something a little bit cool to feel better". P7 commented that colder temperatures further reduced feelings of valence in negative images, "When I chose err cooler it gave me a stronger sense of sadness". According to participant P6: "If its happiness, it $\left(35^{\circ} \mathrm{C}\right)$ makes the feeling a little bit strong with warm temperature". Even though $26^{\circ} \mathrm{C}$ cooling temperature intensity has been shown to increase feelings of valence in negative (low valence) images [2], five out of ten participants still selected it to further reduce feelings of valence in category 2 (negative) images, as the two other temperature intensities $\left(29^{\circ} \mathrm{C} \mathrm{\&} 35^{\circ} \mathrm{C}\right)$ were found more suitable for generally increasing valence in all image emotion categories. P3 expressed their desire for higher intensity temperatures to amplify the feeling of displeasure they wish to communicate in some of the category 2 (negative) images they provided. "Let's say there were just one or two pictures that if there were higher temperatures I would have picked them to really show how I really felt about those pictures". These results show in the creation of augmented images, $26^{\circ} \mathrm{C}$ and $38^{\circ} \mathrm{C}$ stimuli intensities were used to further reduce valence in low valence images, while $29^{\circ} \mathrm{C}$ and $35^{\circ} \mathrm{C}$ intensities were used to increase valence in all image emotion categories

\subsection{Evoking Emotion}

After each participant perceived the augmented image they created at the end of the study, they all agreed the thermal augmentation chosen for each image sufficiently influenced the emotions they wanted in the images and showed confidence it would have the same effect on perceivers. P5 commented that "Yes, for example, one of them I remember it was outside and it was not very cold but I wore very warm clothes, that time I remember I was sweating again this time I chose very warm temperature $\left(38^{\circ} \mathrm{C}\right)$ because I felt warm when I saw the picture (augmented picture) during the slideshow, with me wearing a lot of clothes it reminds me". In the comment by P5 in the previous sentence, the picture was a positive picture when viewed on its own, however the participant chose to reduce the valence in the image using $38^{\circ} \mathrm{C}$ warming temperature. All the participants also thought that using thermal stimuli augmentation could be very effective in evoking different emotions when perceived. Participants P3, P5 and P6 had the following to say P3: "It's basically a very cool way of displaying ones emotion, as in even without the pictures, just placing my hand on the device I could, if let's say I was a blind person I would have been able to tell if what I was supposed to view is a pleasant or unpleasant picture which is a very cool way of going about things". P6: "In fact it's really nice when you feel it, it's like more than just one emotion involved I would say when you have the temperature and you see something sad and feel something cold, it kind of emphasizes your emotions in some sense". P5: "No it was very new actually, very new and exciting for me". Assessing how satisfied participants were 
Using Thermal Stimuli to Enhance Photo-sharing in Social Media:9 • M. Akazue et al.

with the emotive effects of their augmented images shows that all participants felt the augmented images created would effectively evoke the emotions they intend in perceivers.

\subsection{Thermal Stimuli Combinations Selected}

Table 2 shows the proportion of each thermal stimuli combination selected by participants for category 1 (positive) and category 2 (negative) images. The proportion of each thermal combination selected to augment category 1 images was fairly even. This indicates that varying thermal stimuli combinations were used to influence varying emotions in the category 1 images, which is consistent with interview results that shows majority of augmented image creators as wanting to re-create the emotive experiences they had while capturing the images. Achieving this requires selecting varying thermal stimuli combinations designed to amplify the specific emotions participants want to communicate in the images augmented. For category 2 images, participants also selected a fairly even number of $26^{\circ} \mathrm{C}$ and $38^{\circ} \mathrm{C}$ changes to further reduce valence in category 2 images, while $29^{\circ} \mathrm{C}$ and $35^{\circ} \mathrm{C}$ changes seem to have been chosen to increase valence in the images, with $29^{\circ} \mathrm{C}$ being considered as more suitable, as shown by the higher proportion of $29^{\circ} \mathrm{C}$ combinations selected for category 2 images (Table 2)

Table 2. Thermal stimuli combinations (in Degree Celsius) used to augment positive and negative images and their frequency of use.

\begin{tabular}{ccc}
\hline $\begin{array}{c}\text { Image } \\
\text { Category }\end{array}$ & $\begin{array}{c}\text { Thermal } \\
\text { Combination }\end{array}$ & Frequency \\
\hline Category 1 & $26^{\circ} \mathrm{C}$ & $22 \%$ \\
Images & $29^{\circ} \mathrm{C}$ & $26 \%$ \\
& $35^{\circ} \mathrm{C}$ & $24 \%$ \\
& $38^{\circ} \mathrm{C}$ & $28 \%$ \\
\hline Category 2 & $26^{\circ} \mathrm{C}$ & $20 \%$ \\
Images & $29^{\circ} \mathrm{C}$ & $46 \%$ \\
& $35^{\circ} \mathrm{C}$ & $17 \%$ \\
& $38^{\circ} \mathrm{C}$ & $17 \%$ \\
\hline
\end{tabular}

\subsection{Results Summary}

Addressing RQ1, qualitative results and Table 2 shows that 6 out of the 10 participant's augmented category 2 images with the intention of further reducing valence in those images, while the remaining 4 participants attempted to use thermal augmentation to increase valence in category 2 images. Participants' decision to further reduce or increase valence in these images seemed to have mainly been based on whether or not they wanted to suppress how unpleasant the images felt or recreate the exact emotional experiences they had when capturing the images. Participants that attempted to reduce valence did so by selecting $6^{\circ} \mathrm{C}$ changes, while $3^{\circ} \mathrm{C}$ cooling change was considered more suitable for increasing valence in category 2 images felt as shown by the higher proportion of $29^{\circ} \mathrm{C}$ combinations selected for category 2 images (Table 2).

Addressing RQ2, a fairly even number of warming/cooling $3^{\circ} \mathrm{C}$ and $6^{\circ} \mathrm{C}$ changes were selected to influence emotions in category 1 images. Participants generally wanted to increase the realism of the images, thus resulting in an increase in perceived valence. Participants who chose $3^{\circ} \mathrm{C}$ changes found them effective for increasing valence, while participants who selected warming/cooling $6^{\circ} \mathrm{C}$ changes for augmentation did so to increase the realism of specific images, and ultimately increase feelings of valence and arousal in such images.

Addressing RQ3, all participants agreed that the thermal stimuli combination chosen for each image influenced the emotion they intended in the images augmented, and were satisfied with the affective effect of the resulting augmented image.

\section{USER STUDY PART-TWO: EVALUATING THE EMOTIONAL PERCEPTION OF AUGMENTED IMAGES SHARED ON SOCIAL MEDIA}

The second part of our user study was designed to allow a different set of users to perceive and rate the augmented images created in part-one. This allowed us to determine whether thermal stimuli augmentation has the effect part-one participants intended them to have on the images when viewed in a social media interface (research question 4). Following the procedure 


\section{Using Thermal Stimuli to Enhance Photo-sharing in Social Media:10 - M. Akazue et al.}

of [2,25], images were displayed for 6 seconds after which they disappeared and 2 rating scales (see Section 5.3, 'Data Gathering') were presented on screen for 15 seconds each (30 seconds total) for participants to evaluate how each augmented image made them feel. Thermal stimuli augmentation was simultaneously presented to the thenar eminence of perceivers while images displayed, and the neutral stimuli combination $\left(32^{\circ} \mathrm{C}\right)$ was presented while rating scales appeared on screen. The augmented image selection process is further discussed in Section 5.1, 'Image Selection and Presentation' below. In the design of the simulated social media interface used to present the augmented images, we considered some of the currently fastest growing social media platforms (Facebook, Instagram, Snapchat etc.). There has been research which shows that photo-sharing platforms that default to ephemerality (e.g. Snapchat) by deleting content shortly after it is viewed by a recipient reduces self-consciousness and encourages playful interaction more than platforms that support permanent image retention such as Facebook [44]. In Snapchat, images display full screen for a specified amount of time (maximum 10 seconds) after which they disappear. Also Instagram and Facebook, which were platforms that only supported permanent retention of images, recently introduced a new feature referred to as Stories. Similar to Snapchat, Instagram and Facebook Stories allows users to share multiple photos and videos that permanently disappear after 24 hours. Therefore, in the design of the interface used to present the augmented images we evaluated, images in this study were presented in an interface that displayed images full screen, after which they disappeared. This is also similar to the procedure adopted by others $[1,2,29,32]$. Participants were informed the augmented images to be rated were personal and social media images shared by different participants

\subsection{Image Selection and Presentation}

Participants were presented with sample internet images augmented with the extreme thermal stimuli combinations $\left(26^{\circ} \mathrm{C} @\right.$ $3^{\circ} \mathrm{C} / \mathrm{s}$ and $38^{\circ} \mathrm{C} @ 3^{\circ} \mathrm{C} / \mathrm{s}$ ) before commencing the study to familiarise them with what to expect in the study. Once participants felt confident enough to proceed with the experiment, the main study commenced. The thenar eminence was first adapted to the neutral stimuli for 1 minute in order to maximise thermal stimuli perception [3,16,17], after which augmented images were randomly presented to participants to evaluate. A total of 100 images (50 augmented and 50 un-augmented) were presented to each participant to perceive and rate. Half of the images in categories 1 (positive images) and 2 (negative images) were randomly selected and presented with neutral stimuli $\left(32^{\circ} \mathrm{C}\right)$, while the other half of the images in both categories were presented with their corresponding thermal augmentation. Evaluating both augmented and un-augmented versions of the images collected in part-one allowed us to determine the effect of thermal stimuli on augmented images and compare this with the effect part-one participants intended the augmented images to have. It also helps us determine whether thermal augmentation had the desired result part-one participants expected it to have on the images when viewed by perceivers. The entire evaluation lasted for approximately 1 hour, 10 minutes; with a 2-minute break halfway through the evaluation. In order to reduce bias in the evaluation, participants were not told about the affective influence thermal stimuli augmentation was designed to have on the images augmented. Instead augmented and non-augmented versions of the images created in part-one of the study were presented for participants to perceive and evaluate. This follows the approach of others $[1,2,16]$. Augmented and un-augmented images were presented in a random order to participants for evaluation.

\subsection{Participants}

The study was conducted in a quiet lab. Twenty participants (12 males, 8 females) between the ages of 18 and 39 volunteered for this study. Participants had a mean age of 27. All participants were presented with a £10 Amazon voucher after the completion of the study.

\subsection{Data Gathering}

Augmented images were rated in 3 emotional dimensions; valence, arousal and dominance using rating scales based on the vector model of emotion "Self-assessment Manikin (SAM)" [9] and an adapted version of the Russell circumplex model "Emotion Wheel" [6]. SAM is a 9-point Likert scale, which has been used in several studies for evaluating emotional experiences $[2,9,16,42]$. A "9" on the valence and arousal scales corresponded to 'negative valence' and 'calm' respectively, while on the dominance scale it corresponded to 'dominant'. SAM also has human-like figures at each point, which helps minimise the confusion a participant may face while trying to figure out the meaning of each of the emotional dimensions being rated (See Fig. 4). Results by Wilson et al. [42] however showed that though the vector model better explains how emotions relate to perceived valence and arousal, it does not entirely do so. 
Using Thermal Stimuli to Enhance Photo-sharing in Social Media:11 • M. Akazue et al.
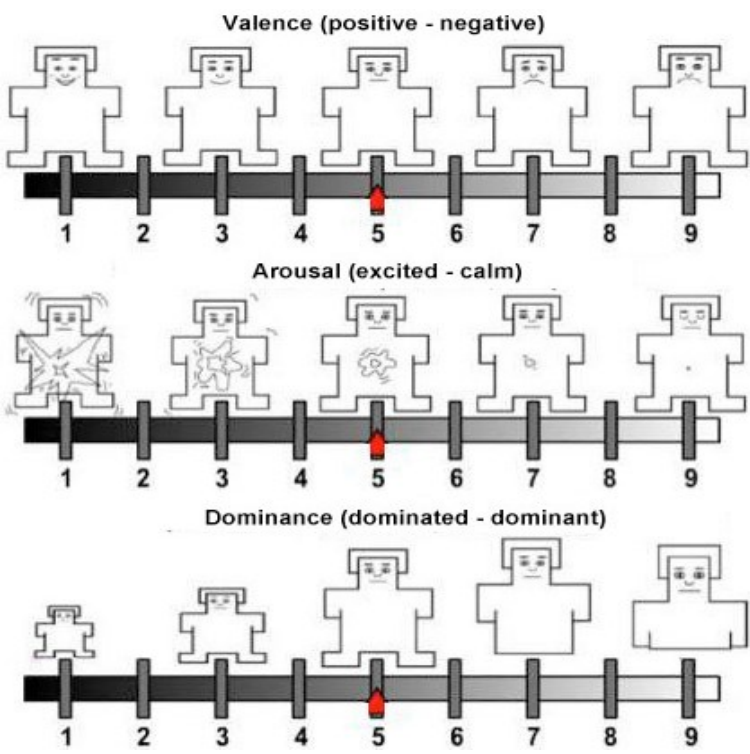

Fig. 4. Self-Assessment Manikin (SAM)

As a result in addition to SAM we also decided to use the Emotion Wheel scale as a supplementary assessment tool. This also follows the procedure of [2]. The Emotion Wheel [6], derived from Russell Circumplex model, is a $0^{\circ}-360^{\circ} \mathrm{cyclic}$ ordinal scale made up of 4 quadrants. The horizontal axis ranges from negative to positive (left to right), and the vertical axis ranges from low-high arousal (bottom to top). The wheel was used as an additional tool to collect self-reported subjective data, this allowed us to assess how participants reported their perception of augmented images (see Fig. 5). Participants were asked to click on the emotion that best described how they felt when they viewed an augmented image. Similar to [2,25], participants were allotted 15 seconds to provide valence, arousal and dominance ratings on SAM and another 15 seconds to provide ratings on the Emotion Wheel following the procedure of [2].

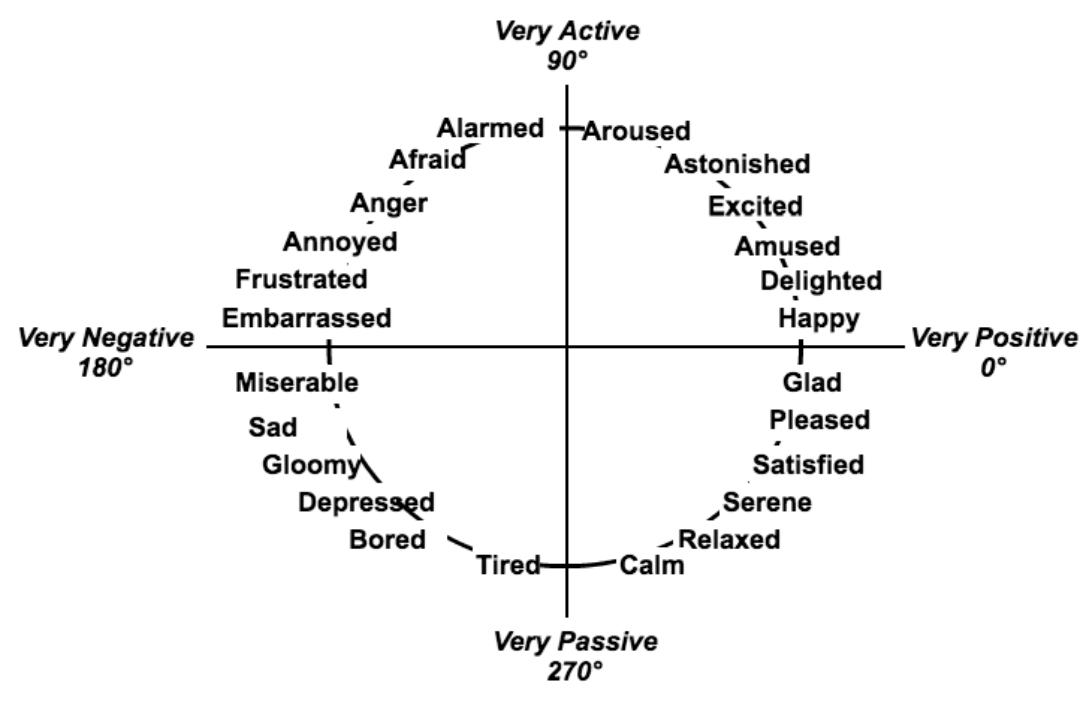

Fig. 5. The Emotion Wheel 
Using Thermal Stimuli to Enhance Photo-sharing in Social Media:12 • M. Akazue et al.

At the beginning of the rating phase, thermal stimuli returned back to neutral $\left(32^{\circ} \mathrm{C}\right)$ for adaptation to occur. The 30 seconds adaptation time between successive stimuli presentation follows the procedure of $[18,19,39,42]$

\subsection{Variables}

This study had a total of 3 independent variables namely; image category, amount of change and direction of change. Image category refers to the positive and negative images supplied by participants in part-one (see Table 2), while amount of change and direction of change are the thermal properties used to create the thermal stimuli combinations in user study partone. The dependent variables were valence, arousal, dominance and Emotion Wheel ratings.

\section{USER STUDY PART-TWO RESULTS}

As data was not normally distributed Friedman Tests and Wilcoxon Sign Rank Tests were used for analysis. Modal values were calculated for the Emotion Wheel variable. This follows the approach adopted by [8] and [2]

\subsection{Image Category}

This independent variable is made up of 2 groups; category 1 images which consisted of positive (high valence/high arousal) images and category 2 images made up of negative (low valence/high arousal) images. As expected, there was a significant difference between the different groups, because the images were grouped based on their inherent emotional properties. As shown in Table 3, pairwise comparison showed that category 1 images had significantly higher ratings of valence ( $\mathrm{z}=-$ 17.393, $p<0.001)$, arousal ( $\mathrm{z}=-3.547, p<0.001)$ and dominance $(\mathrm{z}=-13.298, p<0.001)$, (see Table 3). Modal analysis of results from the emotion wheel, which was designed to be a supplementary data-gathering tool, showed similar results. Category 1 images were rated as 'Happy' while category 2 images were rated as 'Calm'. Based on the positions of the words on the wheel, this indicates category 1 images were perceived to be more pleasant and arousing than category 2 images. This is consistent with results obtained by Akazue et al. [1,2] that showed that images belonging to different emotion categories remained perceptually different after thermal stimuli augmentation.

Table 3. Mean, median in parenthesis and standard deviations of responses relative to the difference in the emotional reactions to image emotion categories $1 \& 2$. Columns with statistically significant differences are marked in blue.

\begin{tabular}{llllc}
\hline Groups & Valence & Arousal & Dominance & $\begin{array}{l}\text { Emotion } \\
\text { Wheel }\end{array}$ \\
\hline Category 1 & $3.22(3.0)$ & $5.02(5.0)$ & $6.64(7.0)$ & 15 \\
Images & $\sigma=1.93$ & $\sigma=2.56$ & $\sigma=2.13$ & $=$ Happy \\
\hline Category 2 & $5.32(5.0)$ & $5.44(5.0)$ & $5.09(5.0)$ & 285 \\
Images & $\sigma=2.55$ & $\sigma=2.59$ & $\sigma=2.68$ & $=$ Calm \\
\hline
\end{tabular}

\subsection{Amount of Change}

This variable is made up of 3 groups which are no change, $3^{\circ} \mathrm{C}$ change and $6^{\circ} \mathrm{C}$ change. A Friedman test showed that the 3 groups did not significantly differ from each other for ratings of arousal $\left(\mathrm{X}^{2}(2)=0.465, \mathrm{p}=0.792\right)$, while a significant difference was found for valence $\left(X^{2}(2)=102.015, p<0.001\right)$, and dominance $\left(X^{2}(2)=50.291, p<0.001\right)$. This led to a pairwise comparison of the groups ( $\mathrm{p}=0.0167$ with a Bonferroni correction). As shown in Table 4 , for valence, $3^{\circ} \mathrm{C}$ changes increased feelings of valence more than when there was no thermal stimuli augmentation $(\mathrm{z}=-4.360, \mathrm{p}<0.001)$, while $6^{\circ} \mathrm{C}$ changes reduced valence more than when there was no thermal stimuli augmentation. $3^{\circ} \mathrm{C}$ changes also significantly increased feelings of valence more than $6^{\circ} \mathrm{C}$ changes $(z=-7.662, p<0.001)$. For dominance, $3^{\circ} \mathrm{C}$ changes significantly increased feelings of dominance more than when there was no thermal stimuli augmentation $(z=-2.9, p=0.004) .6^{\circ} \mathrm{C}$ changes also significantly reduced how dominant participants felt upon perceiving an image more than $3^{\circ} \mathrm{C}$ changes $(\mathrm{z}=-$ 7.662, $\mathrm{p}<0.001)$ and when there was no thermal stimuli augmentation $(\mathrm{z}=-10.505, \mathrm{p}<0.001)$. Modal analysis of the Emotion Wheel showed similar results. $3^{\circ} \mathrm{C}$ changes were rated as 'Happy' (Emotion Wheel rating: 15), increasing valence and arousal more than $6^{\circ} \mathrm{C}$ changes which were rated as 'Calm' (Emotion Wheel rating: 285). This is supported by the Emotion Wheel results from user study part-two which shows that $6^{\circ} \mathrm{C}$ warming and cooling changes significantly reduced 
valence in category 2 (negative) images more than $3^{\circ} \mathrm{C}$ changes and when there was no thermal stimuli augmentation. This result is in slight contrast with findings by Akazue et al. [2] and Wilson et al. [42] which showed $6^{\circ} \mathrm{C}$ cooling changes significantly reduced feelings of valence in low valence (negative) images. This could be because participants in user study part-one actively created augmented images designed to influence specific emotions in perceivers, while Akazue et al. [2] randomly paired images with thermal stimuli combinations in an attempt to identify the effects of individual thermal stimuli combinations on emotions. Also the result reported by Wilson et al. [42] was for when thermal stimuli augmentation is presented on its own.

Results from user study part-two showed that $6^{\circ} \mathrm{C}$ warming and cooling changes reduced valence and arousal in both image categories (positive and negative) as intended, while $3^{\circ} \mathrm{C}$ warming and cooling intensities increased valence and arousal in all images. This contrasts with results from previous work which shows that $6^{\circ} \mathrm{C}$ changes increased feelings of pleasantness, while $3^{\circ} \mathrm{C}$ changes reduced feelings of pleasantness [1,2]. This variance in results could be because participants in part-one of this study chose thermal stimuli augmentation to influence specific emotions in the images augmented, while in the study conducted by $[1,2]$, different thermal stimuli combinations were randomly combined with the images augmented.

Table 4. Mean, median in parenthesis and standard and standard deviations of responses relative to the effect amount of change have on images from categories 1 (positive) and 2 (negative). Columns with statistically significant differences are marked in blue.

\begin{tabular}{ccccc}
\hline Groups & Valence & Arousal & Dominance & $\begin{array}{c}\text { Emotion } \\
\text { Wheel }\end{array}$ \\
No & $4.20(4.0)$ & $5.27(5.0)$ & $5.92(6.0)$ & 285 \\
Change & $\sigma=2.48$ & $\sigma=2.61$ & $\sigma=2.53$ & $=$ Calm \\
$\mathbf{3}^{\circ} \mathbf{C}$ & $3.79(4.0)$ & $5.19(5.0)$ & $6.19(6.0)$ & 15 \\
Change & $\sigma=2.22$ & $\sigma=2.56$ & $\sigma=2.35$ & $=$ Happy \\
$\mathbf{6}^{\circ} \mathbf{C}$ & $4.99(5.0)$ & $5.21(5.0)$ & $5.37(5.0)$ & 285 \\
Change & $\sigma=2.66$ & $\sigma=2.58$ & $\sigma=2.71$ & $=$ Calm \\
\hline
\end{tabular}

\subsubsection{Effect of Amount of Change on Category 1 Images}

Friedman tests of category 1 (positive) images showed that amount of change had no statistically significant effect on the different groups for ratings of valence $\left(X^{2}(2)=1.010, p=0.603\right)$, arousal $\left(X^{2}(2)=0.071, p=0.965\right)$ and dominance $\left(X^{2}(2)=\right.$ $1.179, \mathrm{p}=0.555)$. Modal analysis of the Emotion Wheel ratings also showed that images augmented with $3^{\circ} \mathrm{C}$ changes and images with no thermal stimuli change were perceptually similar; they both made participants feel happy (Emotion Wheel rating: 15$), 6^{\circ} \mathrm{C}$ changes were however rated as having a calming effect (Emotion Wheel rating: 285), reducing feelings of valence and arousal. This is consistent with qualitative results obtained by Akazue et al. [2] that indicated $6^{\circ} \mathrm{C}$ warming changes reduced feelings of pleasantness in high valence images.

\subsubsection{Effect of Amount of Change on Category 2 Images}

Analysis of the effect the amount of change variable has on category 2 (low valence/high arousal) images showed that there was no statistically significant effect between the different groups for ratings of arousal $\left(X^{2}(2)=3.007, p=0.222\right)$. Friedman tests however showed a significant difference for ratings of valence $\left(\mathrm{X}^{2}(2)=8.062, \mathrm{p}=0.018\right)$ and dominance $\left(\mathrm{X}^{2}(2)=\right.$ 50.291, $\mathrm{p}<0.001)$. Pairwise comparison of the 3 groups contained in the amount of change variable $(\mathrm{p}=0.0167$ with a Bonferroni correction) for category 2 (negative) images showed that; images with no thermal augmentation and images augmented with $3^{\circ} \mathrm{C}$ changes were perceptually similar $(\mathrm{z}=-0.684, p=0.494) .6^{\circ} \mathrm{C}$ changes however significantly reduced feelings of valence more than $3^{\circ} \mathrm{C}$ changes $(\mathrm{z}=-3.007, p=0.003)$ and when there was no thermal stimuli augmentation $(\mathrm{z}=$ 4.377, $p<0.001$ ), see Table 5. Emotion Wheel results showed that $6^{\circ} \mathrm{C}$ changes were perceived as 'Sad' (Emotion Wheel rating: 210), significantly reducing valence more than $3^{\circ} \mathrm{C}$ changes which were rated as 'Tired' (Emotion Wheel rating: 255). For dominance, images augmented with $3^{\circ} \mathrm{C}$ intensities and images with no thermal stimuli augmentation $(\mathrm{z}=-1.603, p=$ $0.109)$, as well as images augmented with $6^{\circ} \mathrm{C}$ intensities and images augmented with $3^{\circ} \mathrm{C}$ intensities $(\mathrm{z}=-2.139, p=0.032)$ were perceptually similar. $6^{\circ} \mathrm{C}$ intensities however significantly reduced feelings of dominance more than when there was no thermal stimuli augmentation $(\mathrm{z}=-3.831, p<0.001)$. This is consistent with results by Akazue et al. [2] and Wilson et al. 
[42] which showed $6^{\circ} \mathrm{C}$ cooling changes significantly reduced feelings of valence in low valence (negative) images. The result reported by Wilson et al. [42] was however for when thermal stimuli augmentation is presented on its own.

Table 5. Mean, median in parenthesis and standard deviations of responses relative to the effect amount of change have on category 2 (negative) images. Columns with statistically significant differences are marked in blue.

\begin{tabular}{ccccc}
\hline Groups & Valence & Arousal & Dominance & $\begin{array}{c}\text { Emotion } \\
\text { Wheel }\end{array}$ \\
\hline No & $5.26(5.0)$ & $5.54(5.0)$ & $5.17(5.0)$ & 285 \\
Change & $\sigma=2.56$ & $\sigma=2.61$ & $\sigma=2.67$ & $=$ Calm \\
\hline $3^{\circ} \mathbf{C}$ & $4.81(5.0)$ & $5.46(5.0)$ & $5.45(5.0)$ & 255 \\
Change & $\sigma=2.32$ & $\sigma=2.59$ & $\sigma=2.57$ & $=$ Tired \\
\hline $\mathbf{6}^{\circ} \mathbf{C}$ & $5.75(6.0)$ & $5.26(5.0)$ & $4.71(5.0)$ & 210 \\
Change & $\sigma=2.59$ & $\sigma=2.58$ & $\sigma=2.73$ & $=$ Sad \\
\hline
\end{tabular}

\subsection{Direction of Change}

This independent variable is made up of two groups - warming and cooling. Direction of change had no significant effect on feelings of valence $(\mathrm{z}=-0.221, \mathrm{p}=0.825)$, arousal $(\mathrm{z}=-0.178, \mathrm{p}=0.859)$ and dominance $(\mathrm{z}=-0.787, \mathrm{p}=0.431)$. According to the modal analysis of the Emotion Wheel, warming temperatures were rated as happy (Emotion Wheel rating: 15), increasing valence and arousal more than cooling temperatures which were rated as calm (Emotion Wheel rating: 285).

\subsubsection{Effect of Direction of Change on Category 1 Images}

Pairwise comparison showed that direction of change had no statistically significant effect on category 1 images for ratings of valence $(\mathrm{z}=-0.886, p=0.376)$, arousal $(\mathrm{z}=-0.608, p=0.543)$ and dominance $(\mathrm{z}=-0.631, p=0.528)$. Modal analysis of emotion wheel results showed that images with warming temperatures were perceived as happy (Emotion Wheel rating: 15), while images augmented with cooling temperatures were perceived as relaxing (Emotion Wheel rating: 300). Based on the positions of the words on the wheel, this indicates warming changes increased valence and arousal more than cooling temperatures. Salminen et al. [36] also reported similar results, where warm stimuli were rated to be significantly more arousing and dominant than cool stimuli.

\subsubsection{Effect of Direction of Change on Category 2 Images}

Table 6. Mean, median in parenthesis and standard deviations of responses relative to the effect direction of change have on category 2 (negative) images. Columns with statistically significant differences are marked in blue.

\begin{tabular}{ccccc}
\hline Groups & Valence & Arousal & Dominance & $\begin{array}{c}\text { Emotion } \\
\text { Wheel }\end{array}$ \\
\hline Cooling & $5.31(5.0)$ & $5.28(5.0)$ & $5.01(5.0)$ & 210 \\
& $\sigma=2.58$ & $\sigma=2.58$ & $\sigma=2.71$ & $=$ Sad \\
\hline Warming & $5.70(5.5)$ & $5.60(5.0)$ & $4.98(5.0)$ & 255 \\
& $\sigma=2.28$ & $\sigma=2.60$ & $\sigma=2.61$ & $=$ Tired \\
\hline
\end{tabular}

As shown in Table 6, Pairwise comparison showed that cooling temperatures significantly increased feelings of valence more than warming temperatures $(\mathrm{z}=-3.2, p=0.002)$. No effect was shown for ratings of arousal $(\mathrm{z}=-0.183, p=0.855)$ and dominance $(\mathrm{z}=-0.150, p=0.881)$. A different result was however obtained from the emotion wheel. Modal analysis showed that cooling changes were perceived as sad (Emotion Wheel rating: 210), while warming temperature changes were perceived as tiring (Emotion Wheel rating: 255). Based on the positions of the words on the wheel, this indicates cooling 
Using Thermal Stimuli to Enhance Photo-sharing in Social Media:15 • M. Akazue et al.

changes reduced valence and arousal more than warming changes. This is in line with results by Halvey et al. [16] which showed that warm changes increased feelings of arousal in comparison with cool changes.

\section{DISCUSSION}

\subsection{The Influence of Thermal Stimuli on Emotions in Social Media Images (RQ1, RQ2 and RQ3)}

Participants while creating augmented images in part-one, wanted to use thermal stimuli to increase the realism of the images. Achieving this involves using thermal stimuli augmentation to influence emotions in an image similar to that experienced by the creator of the augmented image. According to comments by P5 (see Section 4.4, 'Evoking Emotions') in user study part-one results, the participants used $6^{\circ} \mathrm{C}$ warming thermal stimuli to reduce valence in category 1 images. This agrees with findings in part-two where emotion wheel results showed that positive (category 1) images, which were perceived as 'Happy' without any thermal stimuli augmentation, were perceived as 'Calm' when augmented with $6^{\circ} \mathrm{C}$ changes. Looking at the positions of 'Happy' and 'Calm' on the Emotion Wheel, it shows that even though images were still positive after thermal augmentation, $6^{\circ} \mathrm{C}$ changes reduced both valence and arousal compared with when there was no change in temperature. Also negative images (category 2) which were perceived as Calm without thermal augmentation were perceived as tiring (slightly lower valence) when augmented with $3^{\circ} \mathrm{C}$ warming stimuli and sad (lower valence and higher arousal) when augmented with $6^{\circ} \mathrm{C}$ cooling stimuli. This indicates that part-one participants may have been successful in communicating some level of realism in the augmented images they created, without being able to noticeably increase the amount of perceived valence in those images, and demonstrates the possibility of using thermal stimuli to influence how an image is perceived.

Although $6^{\circ} \mathrm{C}$ cooling thermal stimuli change was shown to increase feelings of valence in low valence images by Akazue et al. [2], 5 out of the 10 participants we had in part-one selected both $26^{\circ} \mathrm{C}$ and $38^{\circ} \mathrm{C}$ intensities to further reduce feelings of valence in low valence images. According to $\mathrm{P} 3$ in part-one, this could be because $29^{\circ} \mathrm{C} \& 35^{\circ} \mathrm{C}$ were generally perceived suitable for increasing valence in all image categories and the most extreme temperature combinations available were $26^{\circ} \mathrm{C}$ and $38^{\circ} \mathrm{C}$. "There were just 1 or 2 pictures that if they were higher temperatures I would have picked them to really show how I really feel about those pictures". This is supported by the Emotion Wheel results from part-two which shows that $6^{\circ} \mathrm{C}$ warming and cooling changes significantly reduced valence in category 2 (negative) images more than $3^{\circ} \mathrm{C}$ changes and when there was no thermal stimuli augmentation. It is not entirely clear if $6^{\circ} \mathrm{C}$ cooling change was shown to reduce valence in negative images in our study because participants didn't have larger thermal stimuli changes to choose from. More research will be needed to investigate the effect of $6^{\circ} \mathrm{C}$ cooling changes on negative images when larger temperature changes are available for selection.

\subsection{Identifying the Inherent Emotional Property of an Image that is to be augmented with Thermal Stimuli (RQ4)}

The process of correctly identifying the emotional property of an image potentially translates into the ability to correctly choose the thermal stimuli combination capable of influencing an intended emotion in that image. Therefore, we wanted to know how well part-one participants classified the images they provided into emotion categories. Pairwise comparisons of the 2 image categories in part-two showed that images in category 1 (positive) had significantly higher ratings of valence, arousal and dominance than images in category 2 (negative/low valence) before and after thermal stimuli augmentation for ratings of valence, arousal and dominance. This indicates that part-one participants were able to correctly differentiate the images they provided based on valence and arousal. Thus further demonstrating the ability of social media users to create and share augmented images capable of evoking the emotions they intend in a perceiver.

\subsection{The Ability of Augmented Image Creators to Evoke their Intended Emotion in Perceivers (RQ4)}

All participants in part-one upon reviewing their own augmented images agreed thermal stimuli augmentation influenced the emotions intended in the images and felt satisfied with the affective influence of the resulting augmented images. We found that even though each thermal stimuli combination had a label describing the emotions they were designed to influence, 
participants still created augmented images based on the emotion they perceived each thermal stimuli combination evoked in them. P3 stated they could identify the emotions each thermal stimuli combination can enhance or reduce in images just by perceiving the temperature on its own "If let's say I was a blind person I would have been able to tell if what I was supposed to view is a pleasant or unpleasant picture". This is consistent with finding by $[27,35,42,43]$ that shows thermal stimuli augmentation as having the inherent ability to evoke emotions when presented on its own. Results from part-two also showed that augmented images created in part-one had the emotional effect the augmented image creators designed them to have. Part-one participants selected $3^{\circ} \mathrm{C}$ intensities to increase valence and arousal in both positive and negative images and the highest intensity $\left(6^{\circ} \mathrm{C}\right.$ changes) were selected to reduce valence in both positive and negative images. Results from user study part-two showed that $6^{\circ} \mathrm{C}$ warming and cooling changes reduced valence and arousal in both image categories (positive and negative) as intended, while $3^{\circ} \mathrm{C}$ warming and cooling intensities increased valence and arousal in all images. This suggests the ability of social media users to intuitively create augmented images that will evoke the emotion intended in a perceiver when shared on social media.

These findings acts as a foundation, upon which more research investigating the utility of thermal stimuli augmentation in enhancing social media interactions can be built. The results obtained indicate that augmented image creators are able to influence the emotions they intend in the images augmented. This has potential application in various photo sharing contexts. In photo sharing, allowing an image creator to augment the images they create with thermal stimulation provides an additional affective channel, which can potentially be used to communicate more affect to a perceiver. Also allowing social media users to share images augmented with thermal stimuli combinations of choice, has the potential to create more affective social media interactions. In situations where a mobile device such as a tablet device or mobile phone is used to create or consume augmented images, thermal stimuli augmentation can be provided to the fingers similar to [1,29]. When a laptop/desktop computer is used to create or consume augmented images, thermal stimuli augmentation can be presented to the thenar eminence via either a standalone thermal stimuli source similar to the one used by $[1,2,16,18,43]$ or a thermal stimuli source embedded in a control mouse [32].

\section{LIMITATIONS AND FUTURE WORK}

As mentioned in Section 3.4, only male participants responded to the recruitment advertisement in part-one of this study, as a result only male participants participated in the process of creating the augmented images evaluated in user study part-two. Even though males and females have been shown to have similar emotional reactions to images presented on their own $[2,34]$ and thermal stimulation presented on its own [13], we do believe that an investigation of differences in how males and females create augmented images, whilst beyond the scope of this study, would be interesting future work. Furthermore as mentioned in Section 3 ("USER STUDY PART-ONE"), participants were asked to provide images which were previously self-classified into two emotion categories (images perceived to evoke positive and negative emotions). However, images can also be indexed into different emotional categories based on their low-level features such as texture, colour distribution and shape. Using algorithms to index emotions in images based on the low-level feature of individual images has the potential to allow the full automation of the process of creating augmented images designed to influence specific emotions in people. Future work could focus on determining how low-level image properties contribute to the affective influence of thermal stimuli augmentation and the relationship between individual thermal stimuli parameters and each low-level image feature. We also need to test whether augmented images creators are able to influence the emotions they intend in perceivers in other photo sharing scenarios such as one-to-one direct messaging. Finally the two parts of the study presented in this paper were conducted in a quiet lab indoors. We need to test whether external factors (e.g. ambient temperature, humidity etc.) which has been shown to have an influence on thermal stimulation presented on its own $[18,19]$ has any affective influence on augmented images in other usage environment such as outdoors.

\section{CONCLUSION}

In this paper we presented the first known investigation of how the affective attributes of thermal stimuli could be used to enhance photo-sharing interactions on social media. In part-one of the user study, participants were asked to choose their preferred thermal stimuli augmentations for social media images, while the resulting augmented image was perceived and evaluated by a different set of users in part-two. This was to help us understand the reasons behind pairing images with specific thermal stimuli combinations and the emotions augmented image creators wanted to influence in the images augmented. Results from part-one of the study showed that in the creation of augmented images, participants selected $6^{\circ} \mathrm{C}$ 


\section{Using Thermal Stimuli to Enhance Photo-sharing in Social Media:17 • M. Akazue et al.}

thermal stimuli changes to reduce valence in images and $3^{\circ} \mathrm{C}$ changes to increase valence. Results from part-two of the study showed that participants perceived warming and cooling $3^{\circ} \mathrm{C}$ intensities increased valence and arousal, while $6^{\circ} \mathrm{C}$ thermal stimuli changes reduced feelings of valence and arousal. Thus indicating participants in part-one were able to use thermal stimuli to communicate the emotions they intend in the images augmented. Whether it is for self-expression, identity building, or social interaction, augmenting images shared on social media with thermal stimuli has the potential to provide people with a way of effectively communicating emotions in their online images.

\section{REFERENCES}

[1. Moses Akazue, Martin Halvey, and Lynne Baillie. 2017. Using Thermal Stimuli to Influence Affect in Different Picture Display Sizes. Personal and Ubiquitous Computing: 1-19. https://doi.org/10.1007/s00779-017-1018-0

2. Moses Akazue, Martin Halvey, Lynne Baillie, and Stephen Brewster. 2016. The Effect of Thermal Stimuli on the Emotional Perception of Images Proceedings of the 2016 CHI Conference on Human Factors in Computing Systems - CHI '16: 4401-4412. https://doi.org/10.1145/2858036.2858307 Shimon Akiyama, Katsunari Sato, Yasutoshi Makino, and Takashi Maeno. 2012. Presentation of thermal sensation through preliminary adjustment of adapting skin temperature. In 2012 IEEE Haptics Symposium (HAPTICS), 355-358. https://doi.org/10.1109/HAPTIC.2012.6183814

4. Nazanin Andalibi, Pinar Ozturk, and Andrea Forte. 2015. Depression-related Imagery on Instagram. In Proceedings of the 18th ACM Conference Companion on Computer Supported Cooperative work \& social computing, 231-234.

Lynne Baillie. 2002. The Home Workshop: A Method for Investigating the Home.

Lynne Baillie, Lee Morton, David C. Moffat, and Stephen Uzor. 2010. Capturing the response of players to a location-based game. Personal and Ubiquitous Computing 15, 1: 13-24. https://doi.org/10.1007/s00779-010-0309-5

7. Saeideh Bakhshi, David A Shamma, Lyndon Kennedy, and Eric Gilbert. 2015. Why We Filter Photos and How it Impacts Engagement. In Ninth International AAAI Conference on Web and Social Media.

8. David Beattie, Lynne Baillie, and Martin Halvey. 2015. A Comparison of Artificial Driving Sounds for Automated Vehicles. In Proceedings of the 2015 ACM International Joint Conference on Pervasive and Ubiquitous Computing, 451-462.

9. Margaret M. Bradley and Peter J. Lang. 1994. Measuring emotion: The self-assessment manikin and the semantic differential. Journal of Behavior Therapy and Experimental Psychiatry 25, 1: 49-59. https://doi.org/10.1016/0005-7916(94)90063-9

10. Andrew M. Cox, Paul D. Clough, and Jennifer Marlow. 2008. Flickr: a first look at user behaviour in the context of photography as serious leisure. Information Research 13, 1.

11. Jayson L Dibble and Timothy R Levine. 2013. Sharing Good and Bad News with Friends and Strangers: Reasons for and Communication Behaviors Associated with the MUM Effect. Communication Studies 64, 4: 431-452. https://doi.org/10.1080/10510974.2013.770407

Barney Glaser and Anselm Strrauss. 1967. The Discovery of Grounded Theory: Strategies for Qualitative Research.

L Gray, J Stevens, and L Marks. 1982. Thermal stimulus thresholds: Sources of variability. Physiology \& Behavior 29, 2: 355-360. https://doi.org/10.1016/0031-9384(82)90026-9

14. Stefan Greiner, Jan-niklas Antons, and Ina Wechsung. 2015. Emotional Responses to Thermally Augmented Audiovisual Stimuli. Retrieved from http://i-guide.org/app/Papers/23.pdf

15. Rebecca Gulotta, Hakon Faste, and Jennifer Mankoff. 2012. Curation, Provocation , and Digital Identity: Risks and Motivations for Sharing Provocative Images Online. In CHI '12, 387-390.

16. Martin Halvey, Michael Henderson, SA Brewster, Graham Wilson, and Stephen Hughes. 2012. Augmenting media with thermal stimulation. Haptic and Audio Interaction Design 7468: 91-100. Retrieved February 12, 2014 from http://link.springer.com/chapter/10.1007/978-3-642-32796-4 10

17. Martin Halvey, Graham Wilson, Stephen A. Brewster, and Stephen A. Hughes. 2013. Perception of thermal stimuli for continuous interaction. In CHI '13 Extended Abstracts on Human Factors in Computing Systems on - CHI EA '13, 1587. https://doi.org/10.1145/2468356.2468640

Martin Halvey, Graham Wilson, Stephen Brewster, and Stephen Hughes. 2012 "Baby it's cold outside": The Influence of Ambient Temperature and Humidity on Thermal Feedback. In Proceedings of the 2012 ACM annual conference on Human Factors in Computing Systems - CHI'12, 715-724. https://doi.org/10.1145/2207676.2207779

19. Martin Halvey, Graham Wilson, Yolanda Vazquez-Alvarez, Stephen A. Brewster, and Stephen A. Hughes. 2011. The Effect of Clothing on Thermal Feedback Perception. In Proceedings of the 13th international conference on multimodal interfaces - ICMI '11, 217-220. https://doi.org/10.1145/2070481.2070519

20. Nancy A. Van House. 2007. Flickr and public image-sharing. In CHI'07 extended abstracts on Human factors in computing systems - CHI '07, 2717-2722. https://doi.org/10.1145/1240866.1241068

21. C.J. Hutto, Sarita Yardi, and Eric Gilbert. 2013. A longitudinal study of follow predictors on twitter. Proceedings of the SIGCHI Conference on Human Factors in Computing Systems - CHI '13: 821. https://doi.org/10.1145/2470654.2470771

22. L.A. Jones and M. Berris. 2002. The psychophysics of temperature perception and thermal-interface design. In Proceedings 10th Symposium on Haptic Interfaces for Virtual Environment and Teleoperator Systems. HAPTICS 2002, 137-142. https://doi.org/10.1109/HAPTIC.2002.998951

23. Dan R. Kenshalo, Charles E. Holmes, and Paul B. Wood. 1968. Warm and cool thresholds as a function of rate of stimulus temperature change. Perception \& Psychophysics 3, 2: 81-84. https://doi.org/10.3758/BF03212769

24. Dan R. Kenshalo and Harley A. Scott Jr. 1966. Temporal course of thermal adaptation. Science 151, 3714: 1095-1096.

25. Peter J Lang, Margaret M Bradley, and Bruce N Cuthbert. 2008. International Affective Picture System (IAPS): Affective ratings of pictures and instruction manual. Technical Report A-8. University of Florida, Gainesville, FL.

Eunji Lee, Jung-Ah Lee, Jang Ho Moon, and Yongjun Sung. 2015. Pictures Speak Louder than Words: Motivations for Using Instagram. Cyberpsychology, Behavior, and Social Networking 18, 9: 552-556. https://doi.org/10.1089/cyber.2015.0157

27. Wonjun Lee and Youn Kyung Lim. 2012. Explorative research on the heat as an expression medium: Focused on interpersonal communication Personal and Ubiquitous Computing 16, 8: 1039-1049. https://doi.org/10.1007/s00779-011-0424-y

28. Clifton Lin and Haakon Faste. 2012. Photographic Social Media: A Framework For Design. Proceedings of the Designing Interactive Systems Conference on - DIS '12: 244-247. https://doi.org/10.1145/2317956.2317994

29. Markus Löchtefeld, Nadine Lautemann, Sven Gehring, and Antonio Krüger. 2014. AmbiPad: Enriching Mobile DIgital Media with Ambient Feedback. In Proceedings of the 16th international conference on Human-computer interaction with mobile devices \& services - MobileHCI '14, 295-298. https://doi.org/10.1145/2628363.2628395

30. W.J.T. Mitchell. 2005. What Do Pictures Want? The Lives and Loves of Images. University of Chicago Press.

ACM PACM Interact. Mob. Wearable Ubiquitous Technol., Vol. XX, No. XX, Article XX. Publication date: Month YYYY. 


\section{Using Thermal Stimuli to Enhance Photo-sharing in Social Media:18 • M. Akazue et al.}

31. Kimberlee Morrison. 2015. Snapchat is the fastest growing social network. Retrieved June 28, 2016 from http://www.adweek.com/socialtimes/snapchat-is-the-fastest-growing-social-network-infographic/624116

32. Mutsuhiro Nakashige, Minoru Kobayashi, Yuriko Suzuki, Hidekazu Tamaki, and Suguru Higashino. 2009. "Hiya-Atsu" Media: Augmenting Digital Media with Temperature. In Proceedings of the 27th international conference extended abstracts on Human factors in computing systems - CHI EA '09, 3181-3186. https://doi.org/10.1145/1520340.1520453

33. James A. Russell. 1980. A circumplex model of affect. Journal of Personality and Social Psychology 39, 6: 1161-1178.

34. Dean Sabatinelli, C a Tobias Flaisch, Margaret M Bradley, Je R Fitzsimmons, and Peter J Lang. 2004. Affective picture perception: gender differences in visual cortex ? Neuroreport 15, 7: 1109-1112. https://doi.org/10.1097/01.wnr.0000126558.41468.3d

35. Katri Salminen, Veikko Surakka, Raisamo Jukka, Janis Lylykangas, Roope Raisamo, and Kalle Makela. 2013. Cold or Hot? How Thermal Stimuli Are Related to Human Emotional System? In International Workshop on Haptic and Audio Interaction Design., 20-29.

36. Katri Salminen, Veikko Surakka, Jukka Raisamo, Jani Lylykangas, Johannes Pystynen, Roope Raisamo, Kalle Mäkelä, and Teemu Ahmaniemi. 2011. Emotional responses to thermal stimuli. In Proceedings of the 13th international conference on multimodal interfaces - ICMI'11, 193-196. https://doi.org/10.1145/2070481.2070513

37. Katja Suhonen, Kaisa Väänänen-Vainio-Mattila, and Kalle Mäkelä. 2012. User experiences and expectations of vibrotactile, thermal and squeeze feedback in interpersonal communication. BCS-HCI '12 Proceedings of the 26th Annual BCS Interaction Specialist Group Conference on People and Computers: 205-214. Retrieved April 2, 2014 from http://dl.acm.org/citation.cfm?id=2377916.2377939

38. Auke Tellegen. 1985. Structures of mood and personality and their relevance to assessing anxiety, with an emphasis on self-report.

39. Graham Wilson, Stephen Brewster, Martin Halvey, and Stephen Hughes. 2012. Thermal Icons: Evaluating Structured Thermal Feedback for Mobile Interaction. In Proceedings of the 14th international conference on Human-computer interaction with mobile devices and services - MobileHCI '12, 309. https://doi.org/10.1145/2371574.2371621

40. Graham Wilson, Stephen Brewster, Martin Halvey, and Stephen Hughes. 2013. Thermal Feedback Identification in a Mobile Environment. Haptic and Audio Interaction Design: 10-19.

41. Graham Wilson, Gavin Davidson, and Stephen Brewster. 2015. In the Heat of the Moment: Subjective Interpretations of Thermal Feedback During Interaction. CHI'15 Proceedings of the 33rd Annual ACM Conference on Human Factors in Computing Systems: $2063-2072$.

42. Graham Wilson, Dobromir Dobrev, and Stephen A Brewster. 2016. Hot Under the Collar: Mapping Thermal Feedback to Dimensional Models of Emotion. Proceedings of the SIGCHI Conference on Human Factors in Computing Systems (CHI'16).

43. Graham Wilson, Martin Halvey, Stephen Brewster, and Stephen Hughes. 2011. Some Like it Hot: Thermal Feedback for Mobile Devices. Proceedings of the SIGCHI conference on Human Factors in Computing Systems. CHI '11. Retrieved February 13, 2014 from http://dl.acm.org/citation.cfm?id=1979316

44. Bin Xu, Pamara Chang, Christopher L Welker, Natalya N Bazarova, and Dan Cosley. 2016. Automatic Archiving versus Default Deletion: What Snapchat Tells Us About Ephemerality in Design. Proceedings of the 19th ACM Conference on Computer-Supported Cooperative Work \& Social Computing - CSCW'16: 1660-1673. https://doi.org/10.1145/2818048.2819948

ACM PACM Interact. Mob. Wearable Ubiquitous Technol., Vol. XX, No. XX, Article XX. Publication date: Month YYYY. 